\title{
EL CUERPO Y SU PAPEL EN LA COGNICIÓN: UNA APROXIMACIÓN FENOMENOLÓGICA AL CUERPO VIVIDO
}

THE BODY AND ITS ROLE IN COGNITION:

A PHENOMENOLOGICAL APPROACH TO THE LIVED BODY

O CORPO E SEU PAPEL NA COGNIÇÃO:

UMA ABORDAGEM FENOMENOLÓGICA DO CORPO VIVIDO

Stee Hernández Cagua ${ }^{1}$

\section{Resumen}

Este artículo busca exponer cómo el cuerpo desempeñajuega un papel fundamental en la cognición. Para mostrar su importancia se reconstruye el análisis fenomenológico sobre el cuerpo que realiza Husserl en Ideas II. Dicha reconstrucción tiene tres momentos: (1) mostrar que el cuerpo es órgano de la percepción y, por ende, es punto cero de orientación, esto es, portador de cinestesias; (2) exponer que el cuerpo es igualmente portador de sensaciones en el sentido de ubiestesias, es decir, que en la percepción de un objeto se percibe a sí mismo; (3) y, finalmente, mostrar que el cuerpo constituye, en gran parte, el encuentro intersubjetivo.

Palabras clave: cinestesias; ubiestesias; empatía; analogía; intersubjetividad

\section{Abstract}

This paper aims to show how the body plays a fundamental role in cognition. In order to show its importance, Husserl's phenomenological analysis of the body in Ideas II is reconstructed. Such reconstruction has three moments: (1) show that the body is an organ of perception and, therefore, it is a zero point of orientation, that is, a carrier of kinesthesia; (2) show that the body is also bearer of sensations in the sense of ubiestesia, that is to say, that it perceives itself in the perception of an object; and, finally, (3) show that the body is, largely, the intersubjective encounter.

Keywords: kinesthesia; ubiestesia; empathy; analogy; intersubjectivity

1 Estudiante de último semestre de Licenciatura en Filosofía de la Universidad Pedagógica Nacional. Miembro del grupo Filosofía y Enseñanza de la Filosofía. 


\section{Resumo}

Este artigo visa expor como o corpo desempenha um papel fundamental na cognição. Para evidenciar sua importância, reconstruímos a análise fenomenológica do corpo de Husserl em Idéias II. Esta reconstrução tem três momentos: (1) evidenciar que o corpo é um órgão de percepção e, portanto, é um ponto zero de orientação, isto é, um portador de cinestesias; (2) evidenciar que o corpo é igualmente portador de sensações no sentido de ubiestesias, ou seja, que na percepção de um objeto se percebe a si mesmo; (3) e, finalmente, evidenciar que o corpo constitui, em grande parte, o encontro intersubjetivo.

Palavras-chave: cinestesias; ubiestesias; empatia; analogia; intersubjetividade

Fecha de recepción: 16 de agosto de 2017

Fecha de aprobación: 9 de julio de 2018

Para citar este artículo:

Hernández, S. (2017). El cuerpo y su papel en la cognición: una aproximación fenomenológica al cuerpo vivido. Lúdica Pedagógica, 26, 49-54. 


\section{EL CUERPO COMO ÓRGANO DE LA PERCEPCIÓN}

Si bien la cosa o el objeto intencional puede ser descrito a partir de esquemas (Husserl, 2014, párr. 12-17), en los que se muestre detalladamente la mayoría de sus "notas" (o propiedades; extensión, textura, sabor, color, etc.) y sus "relaciones causales con otras cosas" (si $x$, entonces $y$ ), dicha descripción presupone una referencia al cuerpo viviente. Así pues, la cosa tiene cierta extensión porque está estrechamente ligada con determinados movimientos oculares; tiene cierta textura porque está estrechamente ligada a movimientos y recorridos de la mano al "palpar", y así sucesivamente. En último término, se puede afirmar que toda "nota que se le adjudica" a una cosa solo puede ser concebida, en principio, por su relación con el cuerpo sintiente:

El cuerpo es, ante todo, el MEDIO DE TODA PERCEPCIÓN; es el ÓRGANO DE LA PERCEPCIÓN; concurre NECESARIAMENTE en toda percepción. En el ver, el ojo está dirigido a lo visto y pasa corriendo sobre las esquinas, las superficies, etc. Al palpar, la mano se desliza sobre los objetos. Moviéndome, acerco el oído para escuchar. La aprehensión perceptiva presupone contenidos de sensación que desempeñan su necesario papel para la constitución de los esquemas y por ende de las apariciones de las cosas reales mismas. (Husserl, 2014, párr. 18, p. 88).

Si bien, en "evidencia inmediata", el objeto se da al sujeto como una unidad, el sujeto, en todo caso, vía el fenomenologizar, puede "regresar" al trasfondo de pasividad y describir cómo cada sentido (vista, oído, tacto, gusto y olfato) arroja sus respectivos datos que se conjugan armónicamente para constituir la identidad (persistencia) del objeto intencional, en este caso, del objeto perceptual. Así, por ejemplo, una secuencia de "notas táctiles" podría coincidir con una secuencia de "notas visuales". Husserl, concretamente, piensa en la posibilidad de "correlacionar" lisura y brillo. De otra parte, se podría pensar en la percepción de un objeto caliente. El "calor" o la "sensación de caliente" no es una propiedad que se dé al sujeto con la mera visualización, sino que, en rigor, es una propiedad que se da en la esfera táctil; pero una vez constituido esto en el trasfondo de pasividad, el sujeto no se ve en la necesidad, al menos en principio, de ir a constatar dicha propiedad (el calor) de nuevo cuando se le presenta el objeto caliente, sino que percibe la cosa como un todo.

En este marco, una cosa se constituye por su relación con el cuerpo. Esta relación produce sensaciones en el cuerpo que pueden ser seguidas en dos direcciones. En una primera dirección, estas sensaciones presentan propiedades o notas de las cosas. En una segunda dirección, existen un conjunto de sensaciones que son "intuidas" o que el sujeto puede "actualizar" en cualquier momento y que contribuyen a constituir la cosa como una unidad de sentido. Por ejemplo, si un sujeto ve un escorzo frontal de una esfera cuyas propiedades es ser brillante y lisa, entonces, en principio, el sujeto "intuye" que el escorzo no dado (por ejemplo, la parte trasera de la esfera) será brillante y lisa. En todo caso, dicha intuición anticipatoria se puede "defraudar".

De otra parte, que el cuerpo sea órgano de la percepción implica que el cuerpo sea punto cero de la orientación, pues las cosas son percibidas siempre desde un lugar, dirección o posición. Es imposible que una cosa pueda ser percibida desde un no-lugar. Desde luego, el sujeto en la percepción del objeto puede intuir posibles daciones de este en diferentes direcciones. Esta intuición implica un "si me muevo en determinada dirección $y$, entonces el objeto aparecerá en dicha posición $z$ "; por ejemplo, si el sujeto percibe un pupitre, este aparece a su derecha, izquierda, frente o detrás. Del mismo modo, el sujeto podría "idear" un cambio de posición y con aquel cambio, desde luego, una posición nueva del objeto.

A este respecto, Husserl considera que una descripción del cuerpo como punto cero de la orientación o sistema de los lugares permite entender la constitución del "espacio abstracto" que, desde luego, se encuentra en el trasfondo de pasividad:

Se resuelve así el problema de la forma de la intuición espacial. Ésta no es sensible y, sin embargo, en otro respecto es sensible. Sensiblemente dado está el espacio primario de la intuición (sistema de los lugares), que aún no es el espacio mismo. No sensible y, sin embargo, intuitivo en un nivel superior, es el espacio objetivo, que viene a darse a través de la identificación en el cambio, y exclusivamente, por cierto, en el cambio de orientación libre, el que el sujeto mismo ejecuta. (Husserl, 2014, párr. 18, p. 116). 
En este orden de ideas, el cuerpo sintiente o vivido es definido como órgano de la percepción, lo cual implica, a su vez, que el cuerpo sea considerado punto cero de la orientación, pues el objeto perceptual se constituye en la medida en que el cuerpo lo siente (lo ve, lo palpa, lo oye, lo huele, lo prueba) y dicha constitución siempre se da desde un lugar o desde una posición particular. En este proceso, se constituye la unidad de la experiencia perceptual en la que se da un objeto individuado:

El mundo real se constituye primigeniamente en forma escalonada de tal manera que como estrato inferior se edifica la multiplicidad de las cosas de los sentidos [...] en la unidad de la forma espacial. Ahí las cosas de los sentidos se constituyen en la manera subjetiva de la orientación, y para nosotros [...] de tal manera que una cosa de los sentidos señalada, "CUERPo", está dada como portadora constante del CENTRO DE ORIENTACIÓN. La realización se consuma luego de tal forma que las cosas de los sentidos se vuelven estados de las cosas reales; se constituye el sistema de las contexturas reales. (Husserl, 2014, párr. 18, p. 97).

\section{EL CUERPO COMO PORTADOR DE UBIESTESIAS}

Toda percepción es doble: en el percibir (ante todo en el visual) no solo se dan las propiedades de las cosas, sino que también dichas propiedades son sentidas por el cuerpo. No solamente en la percepción adjudico textura a un objeto, sino que también puedo describir cómo se siente esa textura en el mismo (incluso determinar en qué caso la percepción posiblemente sea anómala). Este carácter doble de la percepción es el que permite concebir al cuerpo como vivo. Por ejemplo, cuando un sujeto palpa la superficie de la mesa, ahí no solamente se da la lisura de la misma, sino que en el palpar, el sujeto "siente" su cuerpo, pues esta acción va acompañada de cierta presión en los dedos o "sensaciones de toque". Otro ejemplo, que trae Husserl a discusión, es cuando un sujeto frota algún dedo de su mano en una superficie plana, y una vez termina, persiste una sensación en el dedo, como un tipo de presión que Husserl denominó sensaciones de toque:
El cuerpo, por ende, se constituye primigeniamente de manera doble: por un lado es cosa física, materia, tiene su extensión, a la cual ingresan sus cualidades reales, la coloración, lisura, dureza, calor, y cuantas otras propiedades similares haya; por otro lado, encuentro en él, y siento en él, y dentro de él: el calor en el dorso de la mano, el frío en los pies, las sensaciones de toque en las puntas de los dedos. (2014, párr. 36, p. 185).

Del mismo modo, el cuerpo tiene "propiocepción". Esta es una propiedad que permite que el sujeto se sienta a sí mismo; por ejemplo, si una persona está sentada con las piernas cruzadas, ella sabe y siente su posición corporal. Asimismo, como lo han definido Zahavi y Gallagher (2008), el cuerpo, muchas veces, funciona como un "esquema corporal", es decir que todas las acciones de un sujeto en un entorno son posibles porque este tiene un saber pre-reflexivo (que se encuentra en el trasfondo de pasividad) sobre su corporalidad. En todo momento, el sujeto esquiva obstáculos, logra moverse con éxito en su entorno, logra determinar un "espacio personal", logra caminar por la calle sin estrellarse con la gente, etc. por dicha conciencia pre-reflexiva. Asimismo, dice Zahavi y Gallagher (2008) que sin esa conciencia del cuerpo, sería imposible que el sujeto se moviera con éxito en su espacio. El caso de IW (Ian Waterman) ilustra la importancia de esta conciencia pre-reflexiva sobre el cuerpo o del esquema corporal en el sentido de que el cuerpo no solamente se puede reducir a un objeto intencional de la conciencia (o a imagen corporal) ${ }^{2}$.

2 Esto se puede ver claramente en patologías en las que intervienen procesos esquemáticos corporales. El caso de IW es un ejemplo dramático. A la edad de 19 años, debido a una enfermedad, perdió todo el sentido del tacto y la propiocepción del cuello para abajo. Poco después del comienzo de su enfermedad, cuando IW intentaba mover una extremidad o el cuerpo entero, podía iniciar el movimiento, pero no tenía ningún control sobre dónde iba a parar la parte móvil. Si intentaba coger algo, las manos le fallaban o golpeaban algo violentamente y, a menos que las vigilase, estas empezaban a "deambular" sin su conocimiento. Sus manos ya no estaban donde IW pensaba que estaban y solo podía localizarlas mediante la visión. La carencia de retroalimentación propioceptiva de IW tiene dos efectos. En primer lugar, la conciencia propioceptiva pre-reflexiva común de su movimiento corporal ya no funciona para IW. En segundo lugar, su sistema esquemático corporal, responsable del control motor, nunca se actualiza, y, en consecuencia, su cuerpo no puede tener el control motor que necesita para realizar acciones como lo hacemos normalmente. Posteriormente, IW aprendió a controlar sus movimientos, pero solo a través de una intensa concentración mental y una vigilancia visual 
En este marco, que el cuerpo del sujeto sea estesiológico es una condición fundamental para consolidar la vida mental del sujeto, porque el cuerpo al ser sintiente se configura como un "punto de transbordo" que traduce estados causales en estados mentales; por ejemplo, el sentir hambre (sensación) tiene poder causal porque "cambia", de cierto modo, la percepción que tiene el sujeto de su entorno y de sus actividades ejecutadas, pues se pueden tornar, en ese momento, tortuosas e, incluso, producir mal humor; pero también el nexo causal de la "sensación hambre" que, en gran parte, permite apercibir la vida mental del sujeto, puede ser transformada en motivación y en voluntad, pues no solo el sujeto puede controlar su impulso inmediato, sino que puede planificar un horario tal que le permita satisfacer esta necesidad, etc. Desde luego, hay que aclarar que, aunque el cuerpo estesiológico es fundamental para apercibir la vida mental del sujeto, el que padece y actúa no es solamente el "cuerpo", sino el sujeto considerado como una unidad psicofísica (como persona).

En este orden de ideas, que el cuerpo sea estesiológico implica, al menos, tres asuntos: (1) que posea sensaciones (ubiestesias) que permite concebir el fenómeno perceptivo más allá que un flujo y recepción de información del medio, (2) que el cuerpo sea un "esquema corporal" o que haya por parte del sujeto una conciencia pre-reflexiva de la corporalidad que le permite desenvolverse en su medio, y (3) que el cuerpo estesiológico sea una condición para apercibir la vida mental del sujeto porque lo constituye como una unidad psicofísica, en la cual hay un nexo causal y motivacional entre entorno, sensaciones y estados mentales.

constante. Es decir, aprendió a confiar en una combinación de la cinestesia visual (esto es, retroalimentación visual acerca de nuestro propio movimiento generado por el propio movimiento a través del entorno) y la percepción visual de los movimientos de las extremidades, lo que le permitió moverse de una manera controlada. Sin embargo, su conciencia de su propio cuerpo queda completamente transformada: es una conciencia reflexiva en lugar de pre-reflexiva. Debe realizar todos los movimientos con atención; incluso el hecho de sentarse en una silla sin caerse requiere atención constante. Solo puede ponerse de pie si mira a sus pies y, si no se queda quieto en un sitio, puede caerse fácilmente si cierra los ojos o si se va la luz. Si estornuda mientras camina, se altera su concentración y podría caerse. El caso de IW demuestra lo mucho que dependemos de nuestra conciencia propioceptiva-cinestésica pre-reflexiva de nuestro movimiento corporal y de los procesos esquemáticos corporales para la realización de la acción (Zahavi y Gallaguer, 2008, pp. 223-224).

\section{EL CUERPO Y LA INTERSUBJETIVIDAD}

El sujeto no solo es un "esquema corporal", sino que también es una "imagen corporal". Estas co-emergen juntas.

Aunque se puedan idear situaciones en la que un sujeto solipsista conozca el mundo, este solo podrá reconocerse como un miembro del mundo porque vive en un entorno compartido. Husserl muestra que, aunque el cuerpo es el órgano de la percepción, paradójicamente también es el obstáculo de la misma (Husserl, 2014, párr. 41). Por ejemplo, existen muchas partes del cuerpo que para el sujeto son prácticamente invisibles (coronilla, espalda, ojos, nariz, etc.) y solo llega a saber de estas porque percibe al otro y supone por intuición que él también las posee.

Del mismo modo, las emociones, disposiciones y los estados mentales en general de los demás son intuidos por sus expresiones corporales. Muchas veces, el sujeto "intuye" que el otro está disgustado, atento, alegre, etc., por el rubor de su rostro, por su mirada o por sus contracciones faciales. Dichas atribuciones son posibles porque hay un contacto corporal con el otro:

Para establecer entre yo y otro una relación de trato, para comunicarle algo, etc., tiene que estar establecida una relación corporal, una conexión corporal a través de procesos físicos. Tengo que ir hacia él y hablarle. El espacio desempeña por ende un gran papel e igualmente el tiempo [...]. Que cuerpo y alma formen una unidad de experiencia propia y gracias a esta unidad lo anímico reciba su sitio en el espacio y el tiempo: en ello consiste la legítima "naturalización de la conciencia". (Husserl, 2014, párr. 46, p. 209).

En todo caso, la relación con el otro pasa por la empatía y la analogía. Existe en un sujeto una predisposición a estar abierto al otro o, como dice Husserl, de que el sujeto pueda alegrarse y padecer con el otro; pero, ese alegrarse o padecer con implica que el sujeto reduzca a esfera de propiedad "las sensaciones" del otro" en un "cómo sí"; por ejemplo, para entender la rabia del otro, el sujeto mediante la imaginación o evocación de circunstancias iguales puede intentar entender la sensación o el estado mental del otro.

Así las cosas, la vida mental no solo es posible porque el hombre es concebido como una unidad psicofísica 
gracias a la propiedad estesiológica del cuerpo (con sus respectivas cinestesias y ubiestesias), sino porque también hay otros en el entorno mundano-vital del sujeto que le notifican de su vida anímica a través de sus expresiones corporales.

\section{CONCLUSIÓN}

Que la corporalidad sea fundamental e imprescindible en la cognición implica mostrar que la vida mental depende del cuerpo. Para mostrar que es posible notificar la vida mental del sujeto a partir del cuerpo, necesariamente se debe mostrar el papel que desempeña el cuerpo como órgano de la percepción, como cuerpo estesiológico y como "cuerpo intersubjetivo". Como órgano de la percepción, se logró mostrar que los estados perceptuales del sujeto siempre son estados que notifican un modo de darse de la cosa con respecto al cuerpo (espacio egocéntrico); como cuerpo estesiológico, se logró mostrar que el hombre se concibe como unidad psicofísica, en donde la causalidad transformada en motivación constituye, en gran parte, la corriente de vivencias(vida mental) del sujeto; finalmente, como "cuerpo intersubjetivo" se logró mostrar que la atribución de estados mentales a un sujeto indudablemente es posible o está anclado a sus expresiones corporales.

\section{REFERENCIAS}

Husserl, E. (2014). Ideas relativas a una fenomenología pura y una filosofía fenomenológica. (Trad. A. Z. Quijano). México: Fondo de Cultura Económica.

Zahavi, D., y Gallagher, H. (2008). La mente fenomenológica. Madrid: Alianza editorial. 